\title{
In silico characterization and comparison of the fruit ripening related beta- amylase (BAM) gene family in banana genome $A$ and $B$
}

\author{
Erdianty Setiabudi ${ }^{1}$, Karlia Meitha $^{1,2}$, Fenny Martha Dwivany ${ }^{1,2,3, *}$ \\ ${ }^{1}$ School of Life Sciences and Technology, Institut Teknologi Bandung, Jl. Ganesa 10, Bandung 40132, Indonesia \\ ${ }^{2}$ Indonesia Biodiversity and Biogeography Research Institute (INABIG), J. Cimanuk 6, Bandung 40132, Indonesia \\ ${ }^{3}$ Bioscience and Biotechnology Research Center, Institut Teknologi Bandung, J. Ganesa 10, Bandung 40132, Indonesia \\ *Corresponding author: fenny@sith.itb.ac.id
}

SUBMITTED 7 April 2021 REVISED 20 July 2021 ACCEPTED 10 August 2021

\begin{abstract}
Banana is one of the most important commodities for maintaining global food security. Primary metabolic processes during the ripening of banana greatly affect post-harvest quality, particularly in starch metabolism. The betaamylase (BAM) gene family is known as a group of genes that plays an important role in starch metabolism regulation. In this study, we focused on the characterization and comparative analysis of the BAM gene family in DH Pahang and Pisang Klutuk Wulung (PKW) varieties, these being the AA and BB genomes, respectively. The sequences of BAM gene family were retrieved from the database of Musa acuminata 'DH Pahang' and Musa balbisiana 'PKW' genome, then structural and functional characterization was performed, followed by identification of cis-acting elements in the BAM promoter regions. The results showed that the BAM gene family structure was relatively conserved in both genomes, and a putative BAM11 gene was found, the function of which has not been studied in other plants. Cis-acting element analysis showed that they were distinct in the copy number and types of elements that were responsive to various phytohormones. This study suggested that the BAM genes involved in ripening are spatiotemporally regulated. However, further functional genomic analysis is required to describe the specific role and regulation of BAM genes during ripening in banana.
\end{abstract}

KEYWORDS A and B genome; BAM; Banana; Fruit Ripening

\section{Introduction}

Banana is one of the most important crops in the world, playing a key role in maintaining global food security and as a source of income for banana-producing countries (FAO 2020). As a climacteric fruit, banana is harvested when the fruit is physiologically mature, and then it will ripen after being picked from the plant (Dwivany et al. 2016). The common ripening treatment is that mature green bananas are treated with ethylene to accelerate the ripening processes by inducing changes in primary metabolisms (Pathak et al. 2018). The process of pulp softening and sweetening is very important in determining the quality of banana fruit, and is mainly determined by starch degradation. Unfortunately, the process of starch degradation in banana which has high starch content, is still poorly understood, even though this process is also responsible for providing energy for other metabolic processes during ripening (Cordenunsi-Lysenko et al. 2019).

Degradation of starch granules in amyloplasts during ripening involves various hydrolase enzymes. According to Nascimento et al. (2006), $\beta$-amylase (BAM) is vital in the process of total starch degradation because of its role at the final stage of starch degradation and ability to cleave starch chains at a specific position to produce the final product maltose. Furthermore, increased expression of $B A M$ genes is known to correlate with decreased starch content during banana ripening, and vice versa, supported by many studies (Nascimento et al. 2006; Jourda et al. 2016; Miao et al. 2016; Xiao et al. 2018; CordenunsiLysenko et al. 2019). Studies on the BAM genes in bananas currently focus on transcriptome profiling and in silico genomic analysis to identify members of the BAM gene family associated with starch mobilization that are regulated by ethylene during fruit ripening. Jourda et al. (2016) identified 13 MaBAM genes through in silico analysis of the Musa acuminata genome, and Xiao et al. (2018) re-identified 11 MaBAM genes from the GenBank and Banana Genome Hub databases.

Currently, the genomic sequences of bananas were obtained from the Musa acuminata 'DH Pahang' (D'hont et al. 2012) and Musa balbisiana 'Pisang Klutuk Wulung' (Davey et al. 2013) varieties. Starchy bananas have a higher starch content than dessert bananas, and is considered as one of the distinctive genome-directed pheno- 
types. Dessert bananas generally belong to the M. acuminata genome (A genome), while the starchy ones are generally characterized by the presence of both $M$. acuminata and $M$. balbisiana genome (A and B genome) in their genomic background (OECD 2010).

Therefore, based on this information, the aim of this study is to identify and compare Beta-amylase (BAM) genes in M. acuminata 'DH Pahang' and M. balbisiana 'Pisang Klutuk Wulung' (PKW), based on the structure of the genes and proteins, as well as to predict cis-acting elements on the gene promoter regions. The results are fundamentals for future research in improving carbohydrate compositions in bananas in relation to nutrition or palatability.

\section{Materials and Methods}

\subsection{Retrieval of the BAM gene family sequences from DH Pahang and PKW database}

$M a B A M$ gene sequences were obtained from the $M$. acuminata 'DH Pahang' v.1 database on the Banana Genome Hub website (https://banana-genome-hub. southgreen.fr/; (Droc et al. 2013), including gene accessions, coding sequences (CDS), and annotated proteins. MaBAM nucleotide sequences were then used to identify the MbBAM genes through BLASTN searches against the $M$. balbisiana PKW v.0 database (https://banana-genome-hub.southgreen.fr/blast; (Droc et al. 2013). The parameter used was a cutoff value of $10^{(-10)}$ and it was performed on the pseudochromosome database. The selected $M b B A M$ gene accessions were the top hits that were considered of their \% identity score, chromosome number, and structural completeness. $M a B A M$ genes used in this study refer to the research of Xiao et al. (2018) who identified 11 MaBAM genes from the GenBank and Banana Genome Hub databases, based on BLASTP searches against Arabidopsis thaliana and Oryza sativa databases available in NCBI Genbank (https://blast.ncbi.nlm.nih.gov/Blast.cgi?PROGRAM= blastpdanPAGE_TYPE=BlastSearchandLINK_LOC= blasthome; (Clark et al. 2016). Further information regarding the dataset is listed in Suppl. Table 1-4.

\subsection{Prediction of the structure of the BAM genes and the protein sequences}

The structural features of the MbBAM genes were predicted and annotated using the FGENESH+ program (http://www.softberry.com/berry.phtml?topic= fgenes_plusdangroup=programsdansubgroup=gfs;

(Solovyev et al. 2006) with the parameter of organismspecific gene-finding was set to be $M$. acuminata or Dwarf Banana. This program combines ab initio and similarity-based approaches to improve its accuracy, due to the use of protein sequences that have high similarities to the targeted nucleotide sequences (Xiong 2006). The $M b B A M$ gene sequences were obtained based on their similarity to the MaBAM gene sequences, therefore
FGENESH+ approaches can be applied in predicting the structure of the $M b B A M$ genes. The reference protein sequences used were $M a B A M$ proteins obtained from the Banana Genome Hub website, then the structural features of all $B A M$ genes were visualized using the Gene Structure Display Server 2.0 (GSDS) program (http://gsds.cbi.pku.edu.cn; (Hu et al. 2015) with queries of genomic sequences and CDS of the $B A M$ genes. The putative product sequences of the $M b B A M$ genes were also obtained using FGENESH+, together with the results of the prediction of the structure of the genes.

\subsection{Similarity snalysis of $B A M$ nucleotide and protein sequences between $A$ and $B$ genome}

$M a B A M$ nucleotide and protein sequences were compared with $M b B A M$ using Pairwise Sequence Alignment method on the EMBOSS Needle website (https://www.eb i.ac.uk/Tools/psa/emboss_needle/; (Madeira et al. 2019) with standard parameters, in order to obtain the similarity percentage between the nucleotide and protein sequence pairs between the two genomes (e.g. MaBAM1 to $M b$ $B A M 1$, and so on). EMBOSS Needle uses the NeedlemanWunsch algorithm to perform alignment so that each sequence comparison has the same length. Each BAM sequence was aligned and analyzed throughout the entire sequence (Xiong 2006).

\subsection{Functional annotations of the BAM gene family}

Putative $M b B A M$ proteins were annotated using the BLASTP program on the NCBI GenBank (https://blast.ncbi.nlm.nih.gov/Blast.cgi?PROGRAM= blastpdanPAGE_TYPE=BlastSearchandLINK_LOC= blasthome; (Clark et al. 2016) against the Reference Proteins database (refseq_protein), followed by the BAM protein domain and motif analysis from both genomes. The BAM protein domains were analyzed using the NCBI Conserved Domain Search program (https://www.ncbi.nlm.nih.gov/Structure/cdd/wrpsb.cgi; (Lu et al. 2020) against the CDD (Conserved Domain Database) v.3. The results of the domain analysis were visualized using IBS (Illustrator for Biological Sequence) software. The BAM protein motifs are analyzed and visualized using the MEME program (http://meme-suite.org; (Bailey et al. 2015) with the 'Site Distribution' parameter set to Zero or One Occurrence Per Sequence (zoops) and the 'Number of Motif' parameter set to 15 motifs per search. The results of the motif analysis are annotated using the InterProScan program (http://www.ebi.ac.uk/interpro/search/sequence/; (Mitchell et al. 2019).

\subsection{Phylogenetic tree construction of the BAM protein family}

Phylogenetic analysis was performed on the BAM protein sequences from M. acuminata 'DH Pahang' and M. balbisiana 'PKW', as well as BAM proteins in A. thaliana and O. sativa from research dataset of Xiao et al. (2018). First, Multiple Sequence Alignment was performed us- 
ing the MUSCLE program (Edgar 2004), and then manual trimming was performed on the alignment results using the BioEdit v.7.2.5 program (Hall 1999) to remove ends with long gaps. The phylogenetic tree was then constructed using the MEGA X program (Kumar et al. 2018), with the Maximum-Likelihood method and the bootstrap value of 1000 replicates.

\subsection{Prediction of Cis-acting elements in the BAM pro- moter regions}

The 2000 bp upstream genomic sequences representing the promoter regions of $M a B A M$ and $M b B A M$ genes were retrieved from genome sequences of $M$. acuminata 'DH Pahang' and M. balbisiana 'PKW' using SnapGene v.5.1 software, then putative cis-acting element prediction was performed on these sequences using the PlantCARE program (http://bioinformatics.psb.ugent.be/webtools/pla ntcare/html; (Lescot et al. 2002).

\section{Results and Discussion}

\subsection{Characteristics of the BAM gene family with simi- larity analysis of nucleotide sequences between $A$ and $B$ genome}

The structure of BAM genes was visualized using the GSDS 2.0 program (http://gsds.cbi.pku.edu.cn; (Hu et al. 2015), thus we obtained a diagram that displays the exon, intron, 5' and 3'UTR structures of 11 BAM gene pairs (Figure 1). When compared between the two genomes, almost all of the BAM gene pairs have similar exon-intron compositions, with a maximum difference of one exon number. However, BAM2, BAM10, and BAM11 gene pairs have less similar exon structures when compared to other pairs. This result is reflected in the similarity of nucleotide sequences between DH Pahang and Pisang Klutuk Wulung (PKW) (Table 1).

Based on these data, it is clear that even after comparing the entire length of the sequence, high percentage of similarity was still obtained. Therefore, it is suggested

TABLE 1 Similarity percentage of nucleotide sequences between the BAM gene pairs in DH Pahang (A genome) and PKW (B genome) using the global alignment method (EMBOSS-Needle).

\begin{tabular}{llll} 
Gene Pairs & Length Difference (bp) & Gaps (\%) & Identity (\%) \\
\hline BAM1 & 1,266 & 15.1 & 83 \\
BAM2 & 1,884 & 51.1 & 47.9 \\
BAM3 & 101 & 6.7 & 91 \\
BAM4 & 272 & 11.2 & 86 \\
BAM5 & 116 & 5.9 & 91.3 \\
BAM6 & 1,711 & 25.7 & 72.7 \\
BAM7 & 275 & 7.5 & 89.9 \\
BAM8 & 284 & 4.9 & 91.3 \\
BAM9 & 261 & 12.3 & 86.1 \\
BAM10 & 2,372 & 76.5 & 23.2 \\
BAM11 & 3,600 & 41.6 & 56.9 \\
\hline
\end{tabular}

that most of the $B A M$ gene pairs have conserved nucleotide sequences between the two genomes. However, BAM2, $B A M 10$, and $B A M 11$ are exceptional, in which they are less similar in terms of the structure and nucleotide sequences. Sequence variation in $B A M$ genes may indicate a genetic evolutionary event between the two genomes.

\subsection{Prediction of the BAM protein domain and motif with similarity analysis of protein sequences be- tween $A$ and $B$ genome}

Domain analysis was performed on the putative BAM proteins, then the results were visualized to obtain the diagram shown in Suppl. Figure 1. The results of the BAM protein domain analysis in DH Pahang and PKW showed that all putative proteins obtained from the two genomes have the Glycoside Hydrolase family 14 (GH14) domain, which is a typical conserved domain of the BAM family. Thus, it has been validated through in silico analysis that all these proteins are BAM proteins. However, there are protein pairs that have a second domain in addition to the GH14 domain, for example, the BAM1 and BAM3 pairs have an additional BZR1 domain (BRASSINAZOLE RESISTANT1). Although it has not been studied in the genus Musa, various studies had been conducted on this protein in the $A$. thaliana. For example, in a comprehensive study by Monroe and Storm (2018), it is stated that AtBAM7 and AtBAM8 also have BZR1 domain. These proteins have two functional units, consisting of the BAM domain (for sugar ligand-binding) and the BZR1 domain (for DNA-binding), where they work synergistically as a transcription activator in brassinosteroid (BR) signaling (Monroe and Storm 2018). BZR1-BAM transcription factors (TFs) possess Nterminal extensions that are not present in other BAM proteins, putative nuclear localization sequences (NLS), a putative basic helix-loop-helix (bHLH) domain with DNAinteracting residues (Glu13 and Arg16), and inactive catalytic residues (Reinhold et al. 2011).

The $\beta$-amylase enzymes typically act as a monomer composed of an $(\alpha / \beta) 8$ barrel with a deep catalytic cleft where two molecules of maltose can bind to it, and it also contains two conserved glutamic acid residues that act as acid and base during hydrolysis (Mikami et al. 1994; Monroe et al. 2018). Compared to the general $\beta$ amylases with monomeric active form, there is AtBAM2 that acts as a tetramer, with a dimer-of-dimers structure and a putative secondary binding site (SBS) for starch. Each dimer consists of two BAM subunits, creating a "starch-binding groove" lined by conserved residues identified as the SBSs, where a starch chain is expected to bind to the deep groove (Monroe et al. 2018).

As for individual BZR1 protein, various researches have been conducted on M. acuminata, for example in a study by Shan et al. (2019), it is stated that the MaBZR1/2 protein acts as a repressor for fruit ripening genes that respond to BR signaling. Therefore, based on these two studies, it is suggested that the BAM1 and BAM3 protein pairs in DH Pahang and PKW also act as a transcription factor for the banana ripening genes, but this assumption 


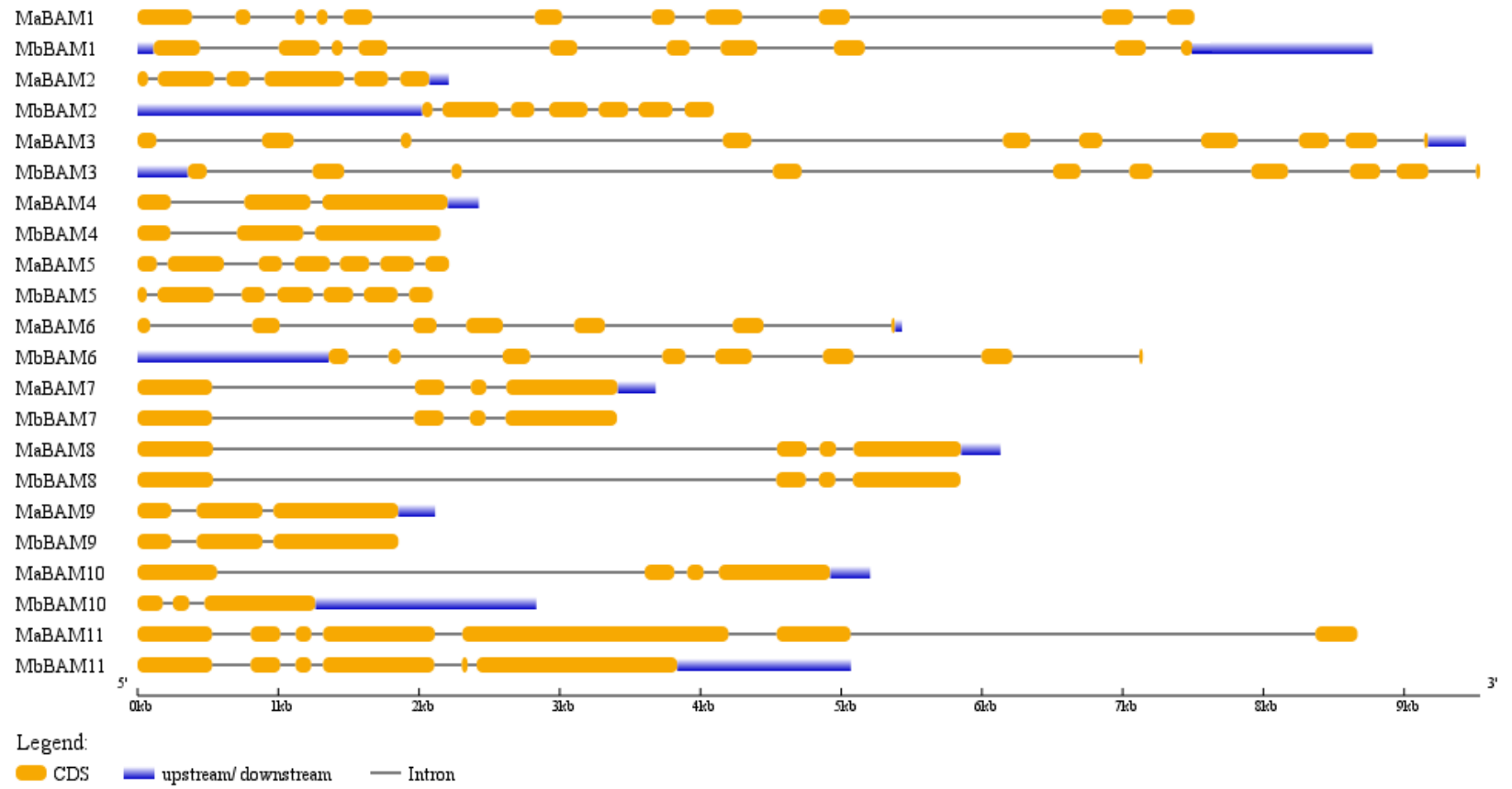

FIGURE 1 The structure of the BAM genes in DH Pahang and PKW with scale description (bp). MaBAM is the name of the BAM genes in A genome, while MbBAM is the name of the BAM genes in $B$ genome. Exons are marked by the yellow shape, while introns are marked by the black line between the exons. The blue box represents the structure of 5'UTR / 3'UTR in the genes.

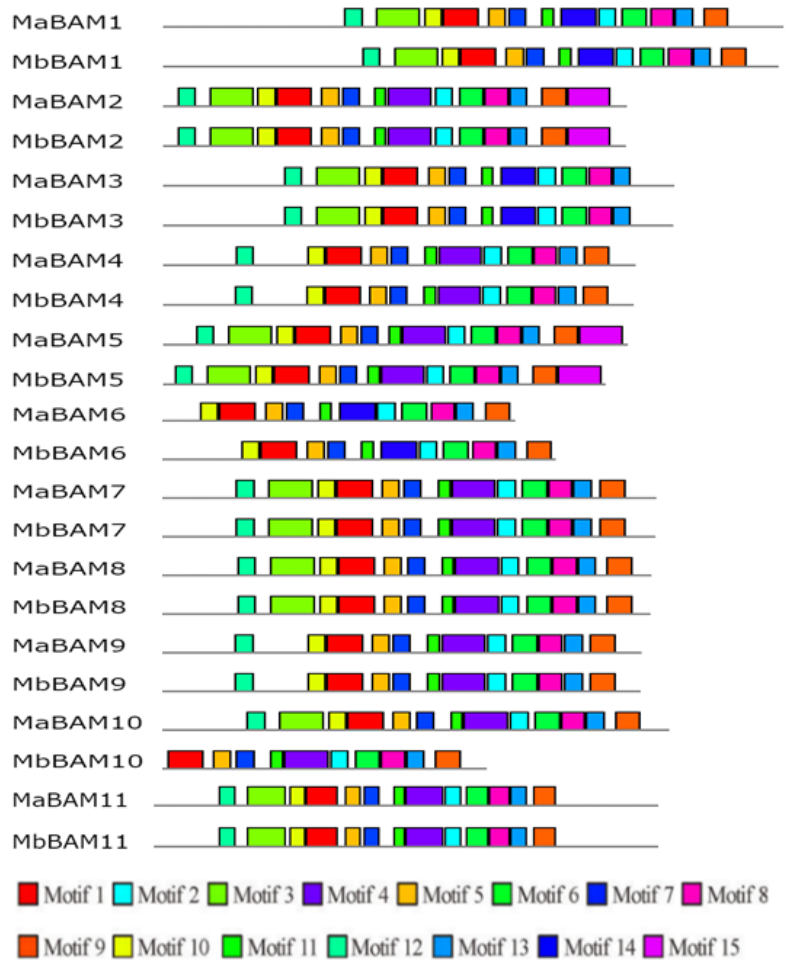

FIGURE 2 The structure of the BAM protein motifs in DH Pahang and PKW with motif numbering.

still has to be proven through wet bench experiments.

The BAM11 protein pair also has a second domain, namely the MRL1 domain, but the research on the MRL1 protein itself is still limited. The MRL1 protein is mainly known to play a role in processing and stabilizing the mRNA of the $r b c L$ gene, thereby increasing Rubisco biogenesis, and ultimately increasing atmospheric $\mathrm{CO}_{2}$ fixation (Johnson et al. 2010). When associated with banana ripening, it is known that high $\mathrm{CO}_{2}$ levels can inhibit the ethylene-dependent ripening process (Larotonda et al. 2008). However, the reason why MRL1 domain was found in the BAM11 protein still cannot be inferred, because the connection between GH14 and MRL1 domains is still not found yet.

Then the BAM protein motif prediction was performed, in which 15 conserved motifs in DH Pahang and PKW were found (Figure 2). Of all motifs, there are 8 motifs annotated as Glycoside Hydrolase family 14 (GH14) domains, and 2 motifs annotated as Glycoside Hydrolase Superfamily, but the rest of them (5 motifs) were not found in the InterPro database. From the InterProScan search, Gene Ontology (GO) annotations were also obtained for the GH14 domain motif, so through in silico analysis, it is suggested that all these putative proteins are involved in polysaccharide catabolism and beta-amylase activity. In addition, when compared between the two genomes, all of these BAM protein pairs have the same motif composition, except for the BAM10 protein pair.

Alignment of BAM proteins in DH Pahang and PKW showed that all BAM isoforms share a huge conserved region around amino acid (aa) 231-676. Moreover, each protein pair from both genomes has really similar amino acid residues in the whole sequences (Suppl. Figure ??2). These results are reflected in the similarity data of the BAM protein sequences between DH Pahang and PKW 


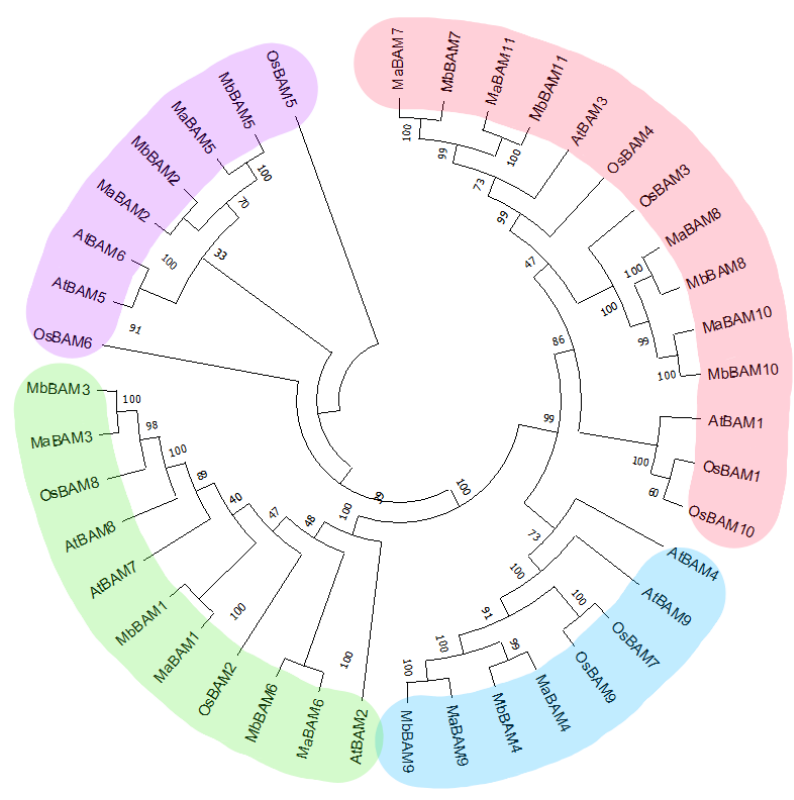

FIGURE 3 Phylogenetic tree of the BAM protein family in M. acuminata, M. balbisiana, O. sativa, and A. thaliana, along with information on taxa grouping represented by different colors.

(Suppl. Table 5), which showed that almost all protein sequence pairs have high similarity (>90\%). All these results may indicate the preservation of structure of BAM proteins within the protein family as well as from both genomes (Miao et al. 2016). However, there is an exception for the BAM10 and BAM11 protein pairs which have lower similarity than the other pairs (range $60-70 \%$ ), and this is thought to be related to the large difference in protein length, and for the BAM10 pair, it is also related to the less conserved motif composition. These results are consistent with the nucleotide analysis discussed earlier. Therefore, it can be concluded that the BAM10 and BAM11 gene in both genomes are less conserved than the other genes when assessed from the structure, although this assumption also has to be proven through wet bench experiments.

\subsection{Phylogenetic analysis of the BAM protein family in M. acuminata, M. balbisiana, A. thaliana, and $\mathrm{O}$. sativa}

When we compared all BAM proteins from DH Pahang and PKW, we obtained four groups with the same motif composition (Suppl. Table 6). These data correspond to the phylogenetic tree containing the BAM protein family members in M. acuminata, M. balbisiana, A. thaliana, and O. sativa (Figure 3 ). In the phylogenetic tree, we obtained four main clades which correspond to the BAM protein groupings mentioned earlier. Therefore, it is suggested that BAM protein pairs with similar sequences join together in the same clade, and considering that amino acid sequences greatly affect the protein function, it can be assumed that each of these clades also contains BAM isoforms among M. acuminata, M. balbisiana, O. sativa, and A. thaliana. Especially for the BAM1 and BAM3 protein pairs in M. acuminata and M. balbisiana, they appear to be in the same clade with the putative isoforms, namely AtBAM7 and AtBAM8.

In this phylogenetic analysis, a homology analysis can be carried out between each pair of BAM10 and BAM11 proteins. It is evident that each BAM10 and BAM11 protein pairs congregate in one closest clade, and this result is sufficient to show that the BAM10 and BAM11 protein pairs are homologous to each other, although the similarity of the sequences is indeed lower than other protein pairs.

\subsection{Analysis of Cis-acting element in the BAM pro- moter regions}

We identified the cis-acting elements in the BAM promoter regions of $\mathrm{A}$ and $\mathrm{B}$ genomes, and we found that 16 motifs are hormone-responsive elements. These motifs are responsive to the hormone ethylene (ERE), auxin (TGAElement, TGA-box, AuxRR-core, AuxRE), gibberellin (GARE, P-box, TATC-box), methyl jasmonate (CGTCAmotif, TCACG-motif), abscisic acid (ABRE, ABRE3a, ABRE4), and salicylic acid (SARE, TCA-Element, TCA). In climacteric fruits, such as bananas, ethylene is the main regulatory factor that controls the fruit ripening process, although there are ethylene-independent processes as well (Pathak et al. 2018). However, it is indicated that the activities of other phytohormones can also interconnect and form signaling networks that coordinate fruit ripening as well, although the mechanisms are still less explored in the genus Musa.

The prior study of cis-acting elements in $B A M$ genes has revealed that the regulation of a $B A M$ gene expression in banana fruit may involve more than one phytohormone, where (Miao et al. 2016) identified cis-acting elements presumably responsive to multiple hormones such as auxin, abscisic acid, and methyl jasmonate in 16 MaBAM genes. For example, for phytohormones which play a role in inducing fruit ripening, there are ABA and methyl jasmonate, and for phytohormones which inhibit fruit ripening, there are auxin, gibberellin, and salicylic acid (Cordenunsi-Lysenko et al. 2019). In this study, we identified elements responsive to these hormones, therefore it is suggested that the $B A M$ genes in DH Pahang and PKW possibly involve regulatory networks of various phytohormones during banana ripening.

In addition, abundance data of cis-acting elements grouped by their function was also collected and then processed into a graph of total cis-acting elements in A and B genome (Figure 4) and a graph of cis-acting element numbers in each $B A M$ gene (Figure 5 ).

Based on Figure 4, it is evident that ethylene and auxin-responsive elements have exactly the same abundance between the two genomes, whereas gibberellin and salicylic acid-responsive elements have similar numbers. This result may indicate that the hormone-responsive elements in the $B A M$ gene family also tend to be conserved between the two genomes. Then based on Figure 5, it is clear that the number of cis-acting elements tends to be dominated by MeJA (methyl jasmonate) and ABA (ab- 


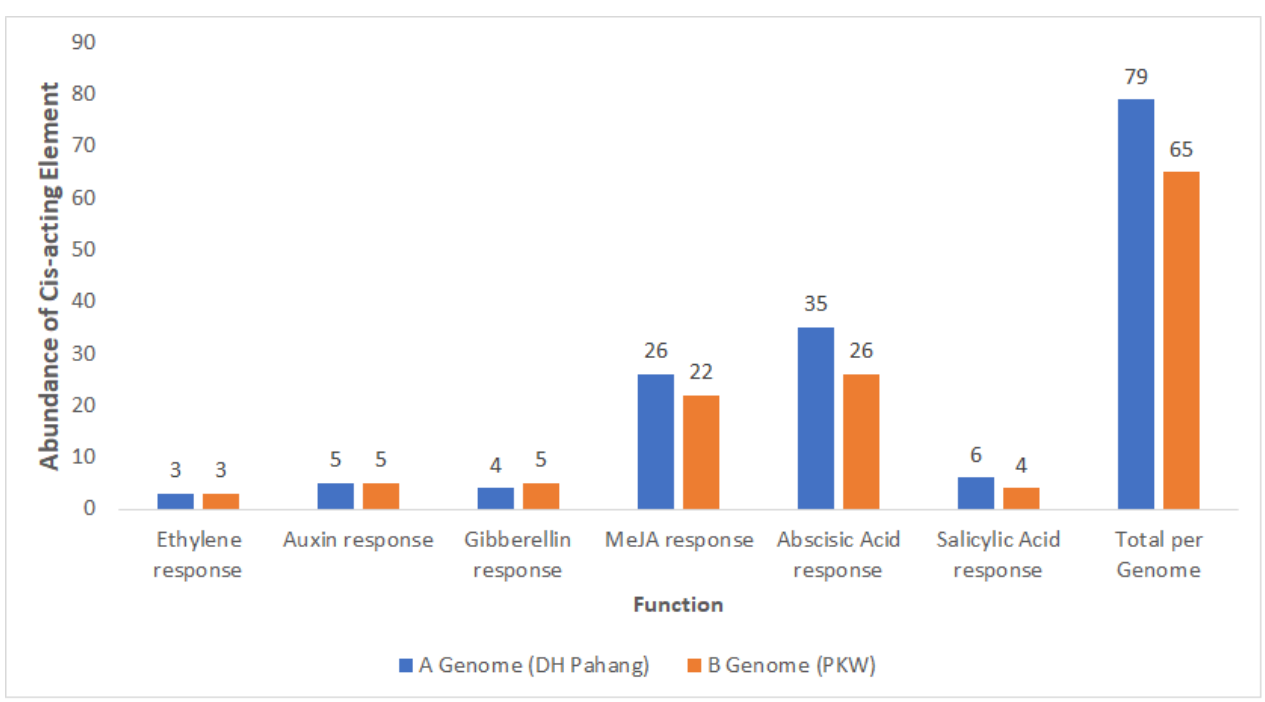

FIGURE 4 Abundance of total cis-acting elements in BAM genes from A genome (DH Pahang) and B genome (PKW) grouped by their functions.

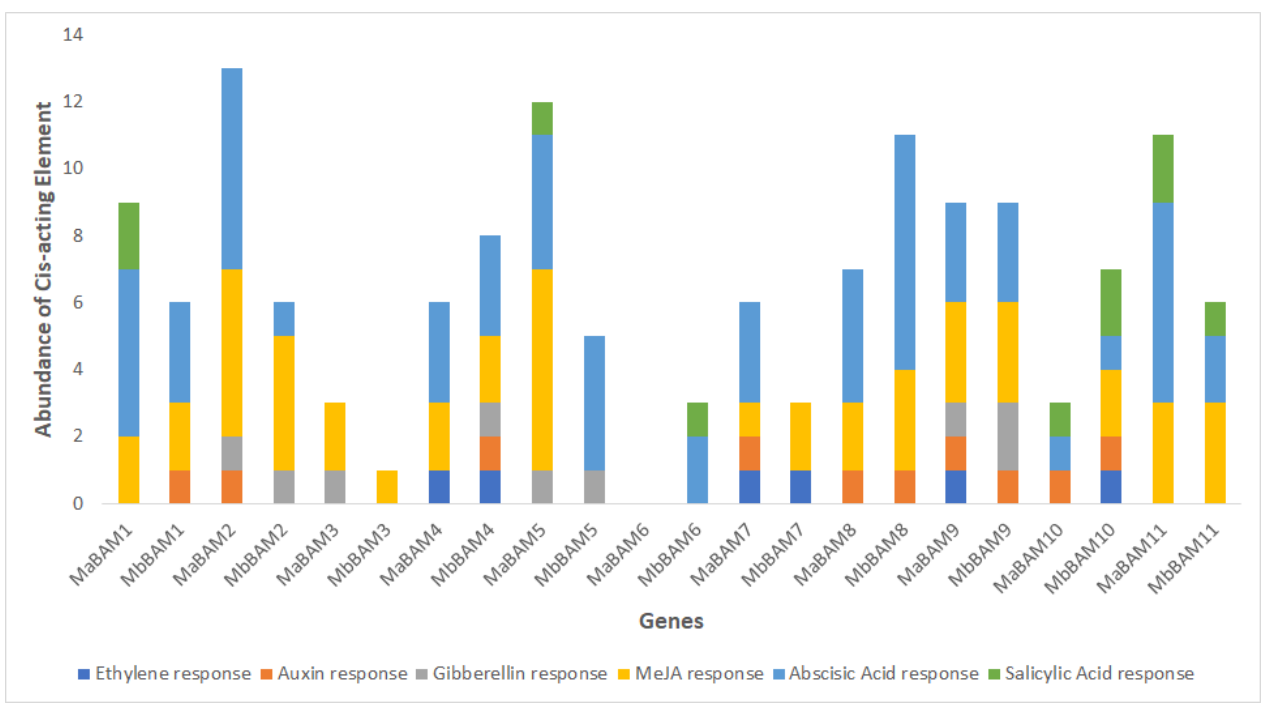

FIGURE 5 Abundance of cis-acting elements in each member of the BAM gene family in DH Pahang and PKW grouped by their functions.

scisic acid)-responsive elements, therefore it is suggested that these two elements are more conserved than the other elements. In addition, there is a slight difference in the copy number and type of cis-acting element between each gene pair, which means that the phytohormones involved may be different between the two genomes. Therefore, these results may indicate differences in $B A M$ genes' regulatory networks between A and B genome. Then considering that the $B A M$ genes are inducible genes, the differences in the cis-acting elements composition may also indicate a difference in transcription time (e.g. at different maturation stages) or location (e.g. in different tissues) of the $B A M$ genes between the two genomes (Biłas et al. 2016).

However, even though there are slight variations, all gene pairs still have similar cis-acting element compositions, therefore the promoter of each gene pair can still be considered as conserved between the two genomes. This cis-acting element study can provide an initial overview of the regulatory network of the BAM gene family in $\mathrm{DH}$ Pahang and PKW during banana fruit ripening. But in fact, for endogenous genes, there are still many endogenous factors that need to be considered, such as the presence of transcription factors and post-transcriptional regulations. Therefore, these cis-acting element data obtained from this in silico analysis may not be fully correlated with the gene expression data from experimental results.

\section{Conclusions}

In conclusion, BAM proteins in M. acuminata ' $\mathrm{DH}$ Pahang' (A genome) and M. balbisiana 'Pisang Klutuk Wulung' (B genome) contain well-conserved structures as characterized by the presence of Glycoside hydrolase family 14 (GH14) domain. It is also suggested that there is a putative BAM11 gene in bananas which role has not been 
studied in other plants, and lastly, there is a slight difference in the composition of the cis-acting elements, indicating differences in the regulatory network of the $B A M$ genes from the two genomes. All results in this study were entirely obtained from in silico analysis, hence validation through functional genomic analysis is suggested for these genes.

\section{Acknowledgments}

This study was supported by Institut Teknologi Bandung and the Indonesian Ministry of Research and Technology.

\section{Authors' contributions}

ES, KM and FMD designed the study, selected and analyzed the data, and wrote the manuscript. All authors have read and approved the final version of the manuscript.

\section{Competing interests}

The authors declare no competing interest.

\section{References}

Bailey T, Johnson J, Grant C, Noble W. 2015. The MEME Suite. Nucleic Acids Res. 43(W1). doi:10.1093/nar/gkv416.

Biłas R, Szafran K, Hnatuszko-Konka K, Kononowicz A. 2016. Cis-regulatory elements used to control gene expression in plants. Plant Cell, Tissue Organ Cult. 127(2):269-287. doi:10.1007/s11240-016-1057-7.

Clark K, Karsch-Mizrachi I, Lipman D, Ostell J, Sayers E. 2016. GenBank. Nucleic Acids Res. 44(D1):67- 72. doi:10.1007/s11240-016-1057-7.

Cordenunsi-Lysenko B, Nascimento J, Castro-Alves V, Purgatto E, Fabi J, Peroni-Okita F. 2019. The Starch Is (Not) Just Another Brick in the Wall: The Primary Metabolism of Sugars During Banana Ripening. Front Plant Sci. 10. doi:10.3389/fpls.2019.00391.

Davey M, Gudimella R, Harikrishna J, Sin L, Khalid N, Keulemans J. 2013. A draft Musa balbisiana genome sequence for molecular genetics in polyploid, interand intra-specific Musa hybrids. BMC Genomics. 14(1):683. doi:10.1186/1471-2164-14-683.

D’hont A, Denoeud F, Aury JM, Baurens FC, Carreel F, Garsmeur O, Noel B, Bocs S, Droc G, Rouard M, et al. 2012. The banana (Musa acuminata) genome and the evolution of monocotyledonous plants. Nature 488(7410):213-217. doi:10.1038/nature11241.

Droc G, Larivière D, Guignon V, Yahiaoui N, This D, Garsmeur O, Bocs S. 2013. The Banana Genome Hub. Database. doi:10.1093/database/bat035.

Dwivany F, Hermawaty D, Esyanti R. 2016. Raja Bulu’ Banana MaACS1 and MaACO1 Gene Expression during Postharvest Storage. Acta Hortic. p. 111-114. doi:10.17660/actahortic.2016.1120.16.
Edgar R. 2004. MUSCLE: multiple sequence alignment with high accuracy and high throughput. Nucleic Acids Res. 32(5):1792-1797. doi:10.1093/nar/gkh340.

FAO. 2020. Medium-term Outlook: Prospects for global production and trade in bananas and tropical fruits 2019 to 2028. Rome: Food and Agriculture Organization of the United Nations.

Hall T. 1999. BioEdit: A user-friendly biological sequence alignment editor and analysis program for Windows 95/98/NT. Nucleic Acids Symp Ser. 41:95-98.

Hu B, Jin J, Guo A, Zhang H, Luo J, Gao G. 2015. GSDS 2.0: An upgraded gene feature visualization server. Bioinformatics. 31(8):1296-1297. doi:10.1093/bioinformatics/btu817.

Johnson X, Wostrikoff K, Finazzi G, Kuras R, Schwarz C, Bujaldon S, Vallon O. 2010. MRL1, a Conserved Pentatricopeptide Repeat Protein, Is Required for Stabilization of rbcL mRNA in Chlamydomonas and Arabidopsis. The Plant Cell. 22(1):234-248. doi:10.1105/tpc.109.066266.

Jourda C, Cardi C, Gibert O, Toro AG, Ricci J, MbéguiéA-Mbéguié D, Yahiaoui N. 2016. Lineage-specific evolutionary histories and regulation of major starch metabolism genes during banana ripening. Front Plant Sci. 7. doi:10.3389/fpls.2016.01778.

Kumar S, Stecher G, Li M, Knyaz C, Tamura K. 2018. MEGA X: Molecular Evolutionary Genetics Analysis across Computing Platforms. Mol Biol Evol. 35(6):1547-1549. doi:10.1093/molbev/msy096.

Larotonda F, Genena A, Dantela D, Soares H, Laurindo J, Moreira R, Ferreira S. 2008. Study of banana (Musa aaa Cavendish cv Nanica) trigger ripening for small scale process. Braz Arch Biol Technol. 51(5):10331047. doi:10.1590/s1516-89132008000500021.

Lescot M, Déhais P, Thijs G, Marchal K, Moreau Y, Peer Y, Rombauts S. 2002. PlantCARE, a database of plant cis-acting regulatory elements and a portal to tools for in silico analysis of promoter sequences. Nucleic Acids Res. 30(1):325-327. doi:10.1093/nar/30.1.325.

Lu S, Wang J, Chitsaz F, Derbyshire M, Geer R, Gonzales N, Marchler-Bauer A. 2020. CDD/SPARCLE: The conserved domain database in 2020. Nucleic Acids Res. 48(D1). doi:10.1093/nar/gkz991.

Madeira F, Park Y, Lee J, Buso N, Gur T, Madhusoodanan N, Lopez R. 2019. The EMBL-EBI search and sequence analysis tools APIs in 2019. Nucleic Acids Res. 47(W1). doi:10.1093/nar/gkz268.

Miao H, Sun P, Miao Y, Liu J, Zhang J, Jia C, Xu B. 2016. Genome-wide identification and expression analysis of the $\beta$-amylase genes strongly associated with fruit development, ripening, and abiotic stress response in two banana cultivars. FASE. 3(4):346. doi:10.15302/j-fase-2016127.

Mikami B, Degano M, Hehre E, Sacchettini J. 1994. Crystal structures of soybean b-amylase reacted with bmaltose and maltal: active site components and their apparent roles in catalysis. Biochemistry. 33:7779- 
7787. doi:10.1021/bi00191a005.

Mitchell A, Attwood T, Babbitt P, Blum M, Bork P, Bridge A, Finn R. 2019. InterPro in 2019: Improving coverage, classification and access to protein sequence annotations. Nucleic Acids Res. 47(D1). doi:10.1093/nar/gky1100.

Monroe J, Pope L, Breault J, Berndsen C, Storm A. 2018. Quaternary structure, salt sensitivity, and allosteric regulation of $\beta$-AMYLASE2 from Arabidopsis thaliana. Front Plant Sci. 9. doi:10.3389/fpls.2018.01176. URL https://doi.org/ 10.3389/fpls.2018.01176.

Monroe J, Storm A. 2018. Review: The Arabidopsis $\beta$-amylase (BAM) gene family: Diversity of form and function. Plant Sci. 276:163-170. doi:10.1016/j.plantsci.2018.08.016.

Nascimento J, Júnior A, Bassinello P, Cordenunsi B, Mainardi J, E P. 2006. Beta-amylase expression and starch degradation during banana ripening. Postharvest Biol Technol. 40. doi:10.1016/j.postharvbio.2005.11.008.

OECD. 2010. Safety Assessment of Transgenic Organisms, Volume 4. Harmonisation of Regulatory Oversight in Biotechnology. doi:10.1787/9789264096158-en.

Pathak S, Sriramulu S, Thandavan S, Jothimani G, Banerjee A, Marotta F. 2018. Enhancement of shelf life of the climacteric fruits: a review on application of CRISPRi technology. Trends Tech Sci Res. 1(2).

Reinhold H, Soyk S, Simková K, Hostettler C, Marafino J, Mainiero S, Vaughan CK, Monroe JD, Zeeman SC. 2011. B-Amylase-Like Proteins Function As Transcription Factors in Arabidopsis, Controlling Shoot Growth and Development. Plant Cell. 23(4):13911403. doi:10.1105/tpc.110.081950.

Shan W, Guo Y, Wei W, Chen J, Lu W, Yuan D, Kuang J. 2019. Banana MaBZR1/2 associate with MaMPK14 to modulate cell wall modifying genes during fruit ripening. Plant Cell Rep. 39(1):35-46. doi:10.1007/s00299-019-02471-5.

Solovyev V, Kosarev P, Seledsov I, Vorobyev D. 2006. Automatic annotation of eukaryotic genes, pseudogenes and promoters. Genome Biol. 7(Suppl 1). doi:10.1186/gb-2006-7-s1-s10.

Xiao Y, Kuang J, Qi X, Ye Y, Wu Z, Chen J, Lu W. 2018. A comprehensive investigation of starch degradation process and identification of a transcriptional activator MabHLH6 during banana fruit ripening. Plant Biotechnol J. 16(1):151-164. doi:10.1111/pbi.12756.

Xiong J. 2006. Essential bioinformatics. Cambridge: Cambridge University Press. 